\title{
Evaluation of Blood Glucose Control Using HbA1c before and after Four Injections of Insulin Analogues in Children with Diabetes Mellitus Type 1
}

\author{
Zahra Razavi $^{1, *}$, Parisa Pouya ${ }^{2}$, Mohammad Ali Saifrabiei ${ }^{3}$, Mohammad Kazem Sabzehei ${ }^{1}$ \\ ${ }^{1}$ Associate Professor, Department of Pediatrics, School of Medicine, Hamadan University of Medical Sciences, Hamadan, \\ Iran \\ 2 Student of Medicine, Hamadan University of Medical Sciences, Hamadan, Iran \\ ${ }^{3}$ Associate Professor, Department of Social Medicine, School of Medicine, Hamadan University of Medical Sciences, \\ Hamadan, Iran
}

* Corresponding Author: Zahra Razavi, Department of Pediatrics, School of Medicine, Hamadan University of Medical Sciences, Hamadan, Iran. Email: razavizahra@yahoo.com.au

Received: 14.05 .2018

Accepted: 14.08 .2018

\section{How to Cite this Article:}

Razavi Z, Pouya P, Saifrabiei MA, Sabzehei MK. Evaluation of Blood Glucose Control Using HbA1c before and after Four Injections of Insulin Analogues in Children with Diabetes Mellitus Type 1. Avicenna J Clin Med. 2018; 25(2): 92-98. DOI: 10.21859/ajcm.25.2.92

\section{Abstract}

Background and Objective: Acceptable control of blood glucose is one of the most important goals of treatment management in diabetic children. An insulin therapy regimen with multiple daily injection or continuous subcutaneous insulin infusion has been admitted as a desirable therapeutic approach in children with diabetes mellitus type 1 . This study was designed to evaluate the effect of intensive insulin therapy on HbA1c as an alternative for the conventional two-injection insulin therapy.

Materials and Methods: This descriptive-cross sectional study was conducted on 100 children and adolescents with diabetes mellitus type 1 at Pediatric Endocrinology Outpatient Clinic of Besat Hospital, Hamadan, Iran in 2017. The present study was conducted on the patients that were previously treated with twice-daily NPH and regular insulin, and then with four injections of insulin Aspart per day before each meal and insulin Glargine at bedtime. Demographic data included: age of onset of diabetes, weight, height, body mass index, duration of diabetes, number of hypoglycemic events, number of episodes of diabetic ketoacidosis (a year before and after the change of insulin regimen), number of insulin injections, type of insulin, injection device, and mean of HbA1c (a year before and after the change of insulin regimen). The data were analyzed using SPSS software (version 16) and appropriate statistical tests.

Results: The mean age of the patients was 11.41 years. Out of 100 subjects, 60 cases (60\%) were female and $75 \%$ were male. In addition, $75 \%$ and $25 \%$ of the cases were urban and rural, respectively. The average body mass index was 19.65. The average number of visits to the clinic and measurement of HbA1c were 3.33 times a year. The mean of $\mathrm{HbA1c}$ before and after four injections per day was 8.4 and 8.1, respectively. There was a statistically significant difference $(\mathrm{P}=0.03)$. The mean reduction was significant $(\mathrm{P}=0.001)$ in subjects that were referred to the clinic and performed HbA1c more than three times a year. The frequency of reported hypoglycemia was 1.4 each year and the difference was not statically significant .There were no differences in terms of hypoglycemia events before and after four injections per day.

Conclusion: The findings of this study confirmed that multiple daily injection of insulin is associated with improved metabolic control of blood glucose. However, HbA1c levels were not in the range of good control.

Keywords: Diabetes Mellitus Type 1, Glycosylated Hemoglobin, Insulin Analogues 


\title{
بررسى وضعيت كنترل قند خون با استفاده از معيار HbA1c قبل و بعد از رذيم جهار نوبت تزريق آنالوكهاى انسولين در كودكان مبتلا به ديابت نوع يك
}

\author{
زهرا رضوى اء*، ير يسا يويا'، محمد على سيف ربيعى ‘، محمد كاظم سبزهاى' \\ ' دانشيار، گَروه كودكان، دانشكده يزشكى، دانشكاه علوم يزشكى همدان، همدان، ايران

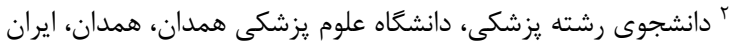

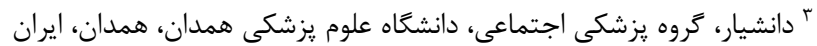 \\ * نويسنده مسئول: زهرا رضوى، گروه كودكان، دانشكده يزشكى، دانشكاه علوم يزشكى همدان، همدان، ايران.
} ايميل: razavizahra@yahoo.com.au

\begin{tabular}{|c|c|}
\hline 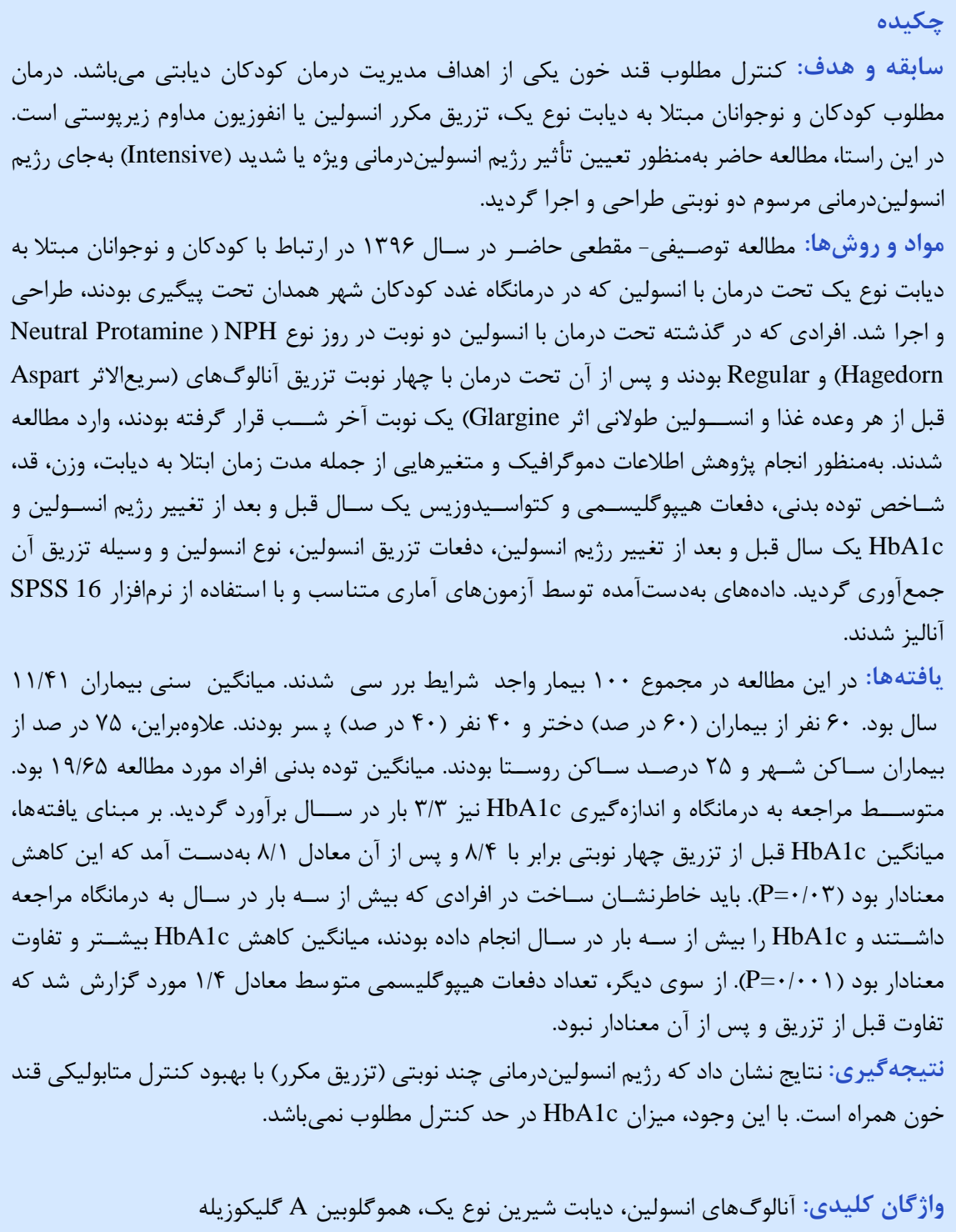 & 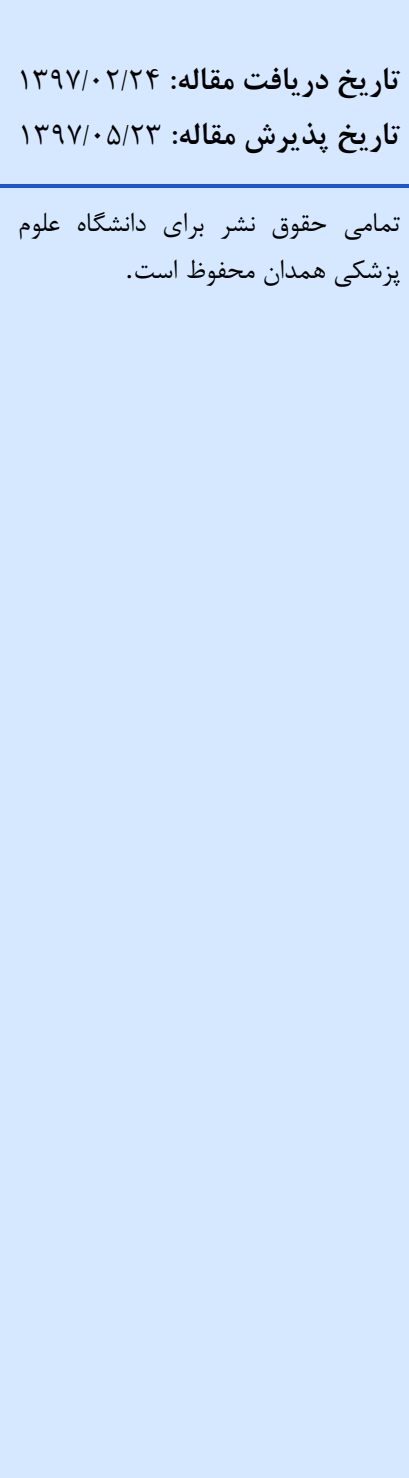 \\
\hline
\end{tabular}


ديخَ، آلبا و همكاران در يك مطالعه كوهورت گذشتنهنگر در مورد

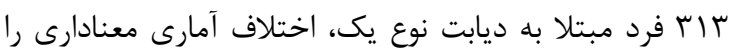
در ميزان كاهش هموكلوبين كليكوزيلهشده بين دريافت كننده

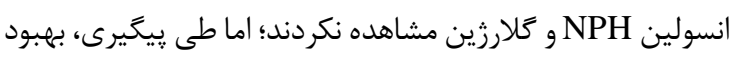

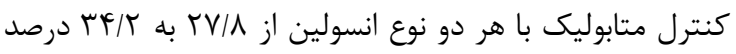

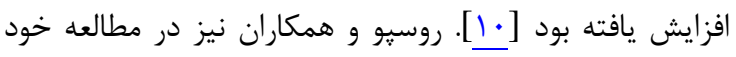
كزارش كردند كه اثرات مفيد تزريق مكرر و هدفترفتن

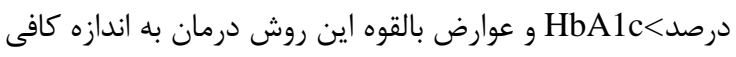

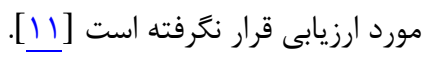

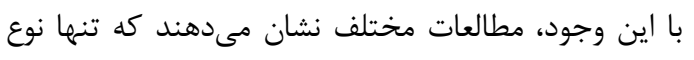

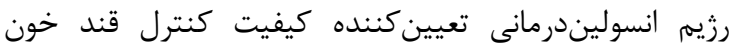

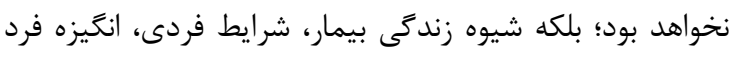

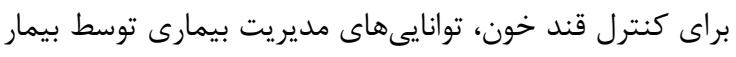

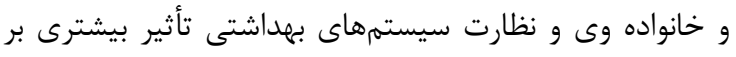

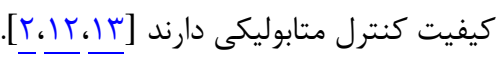

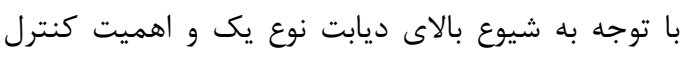

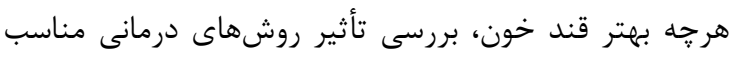

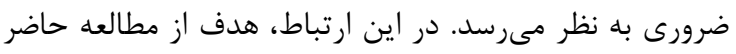

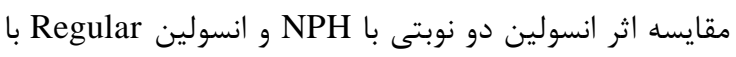

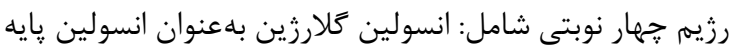

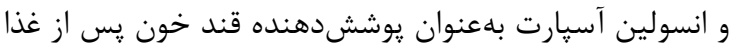

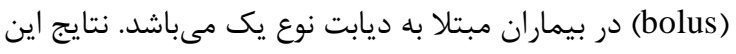

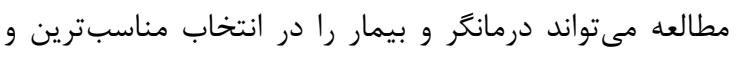

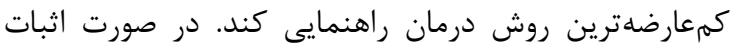

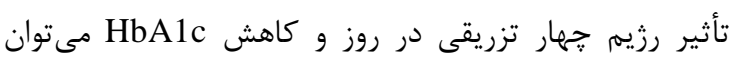

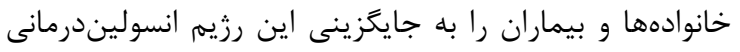

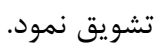

\section{مواد و روشها}

مطالعه توصيفى - مقطعى حاضر يس از تصويب كميته اخلاق دانشعاه علوم يزشكى همدان و دريافت رضايتنامه كتبى آثاهانه

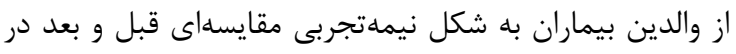

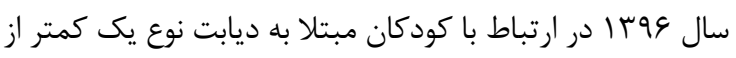

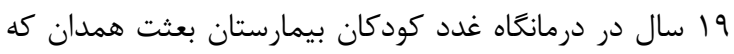
يك مركز ارجاع بيماران ديابتى مىباشد، طراحى و اجرا دران كرديد.

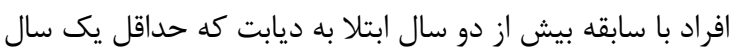

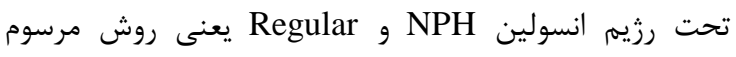
انسوليندرمانى بودند و سيس حداقل به مدت يك رئ سال تحت

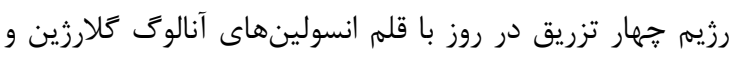

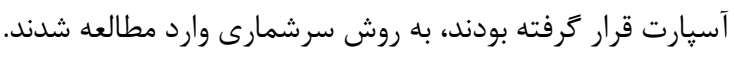

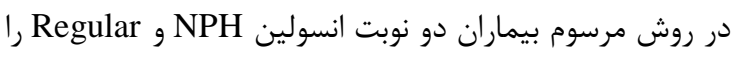

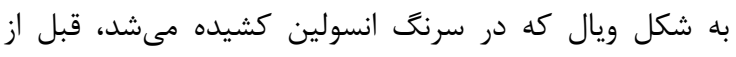

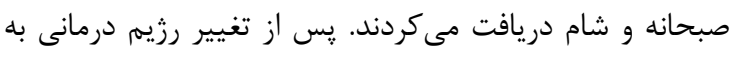

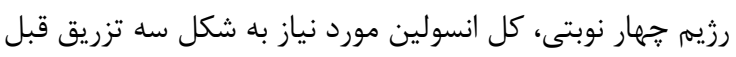

تخريب خودايمنى سلولهاى بتاى جزاير يانكراس مىباشد. نتيجه

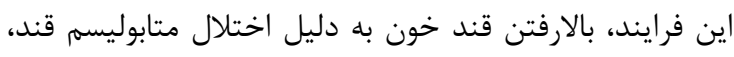
خربى و يروتئين در بدن و بروز عوارض حاد ماد مانند كتواسيدوز

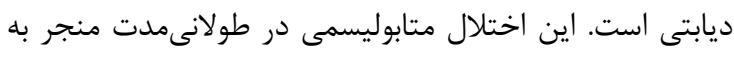

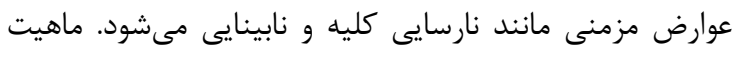

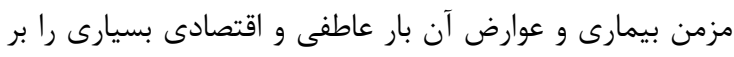

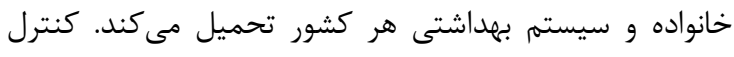

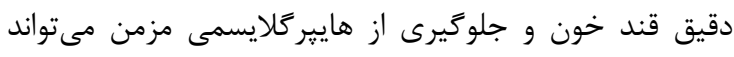

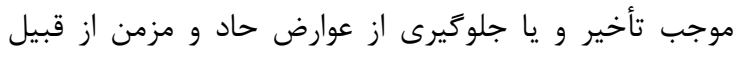

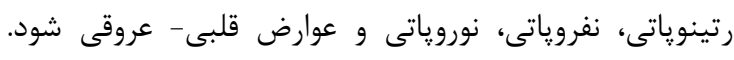

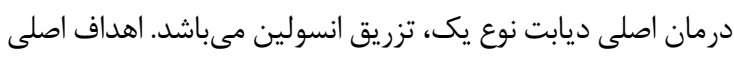

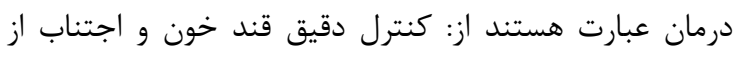

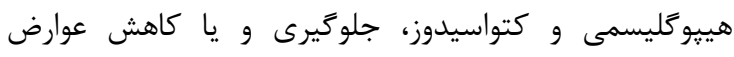

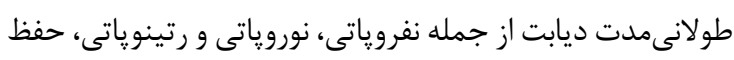
سبك زندكى عادى و روند رشد مناسب. يايش كيفيت كنترل

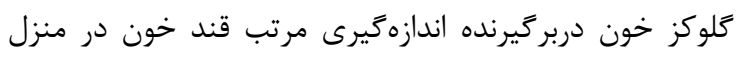

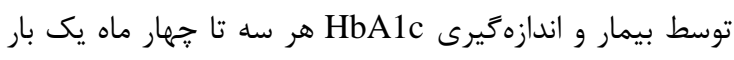

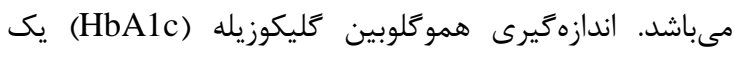
شاخص قابل اعتماد در ارزيابى كنترل سه ماهه قند خوند خون است.

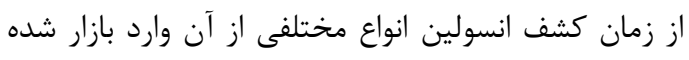

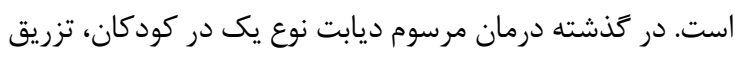

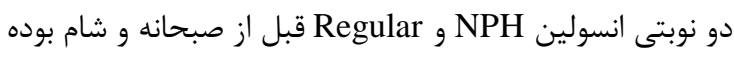
است؛ اما امروزه رزيم درمانى مطلوب توصيهشده براى كودكان،

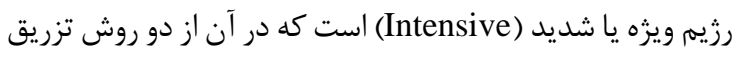

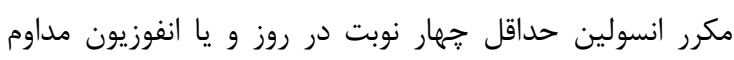

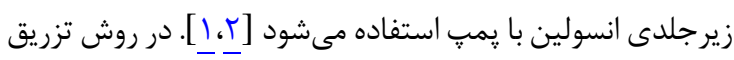

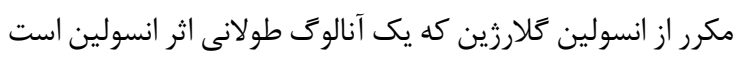

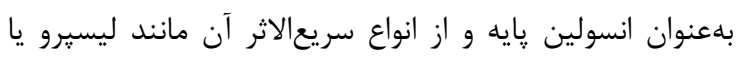
آسيارت قبل از وعدههاى غذايى و يا ميانوعده بلهعنوان بولوز

استفاده مىشود.

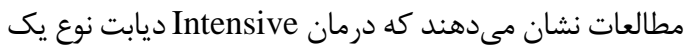

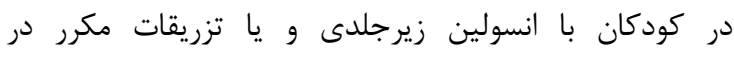
نزديكشدن قند خون به محدوده قابل قبول، يذيرفتهتر از رزينم

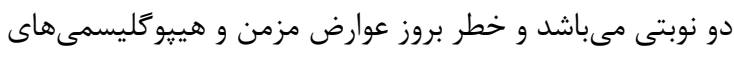

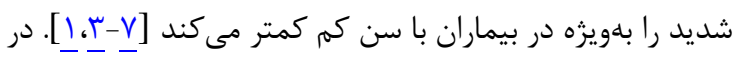

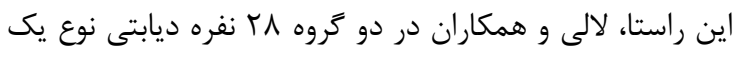

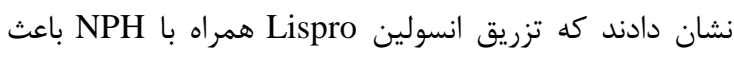

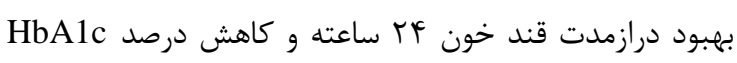

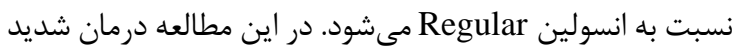

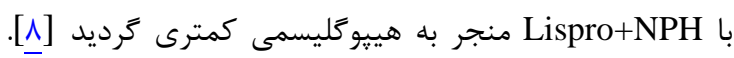
نوردوال و همكاران نيز نشان دادند كه درمان مطلوب و شديد شيديد

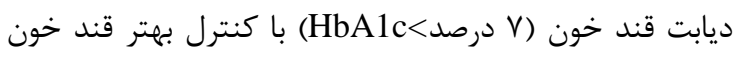

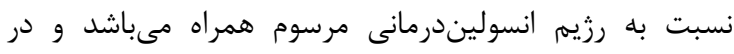

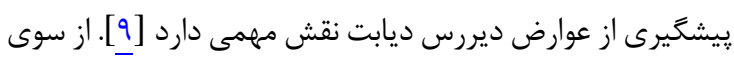


يا كاهش سطح هشيارى همراه بودند، بلعنوان هييو كليسمى

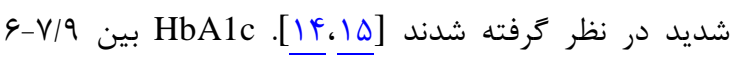

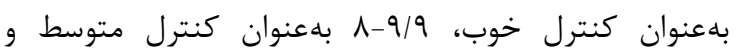

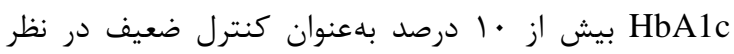
كرفته شد [19]. بايد خاطرنشان ساخت كه افراد فاقد يُيخيرى دورهاى مرتب

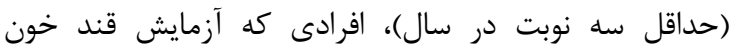

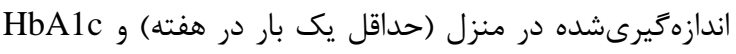

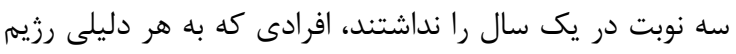

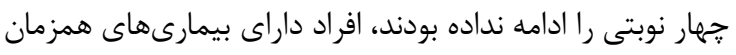
مانند بيمارىهاى كليوى و افراد مصرفكننده داروهاى تأثير گنذار

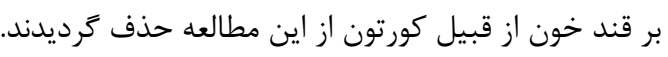

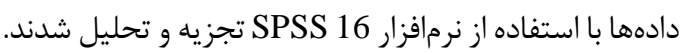

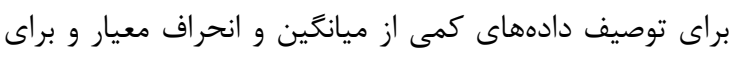

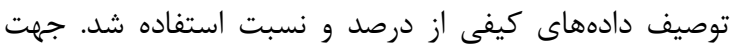

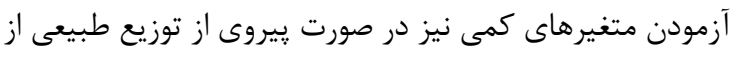

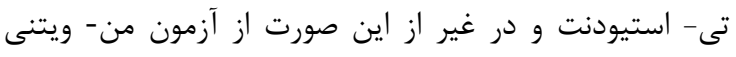

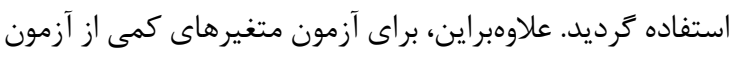

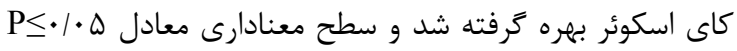

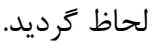

\section{بافته ها}

در اين مطالعه در مجموع · ا بيمار مبتلا به ديابت نوع يك سل

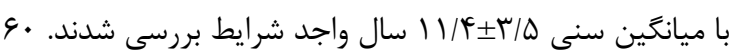

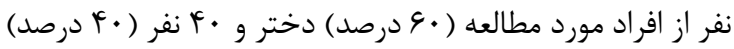

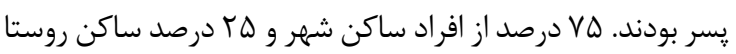

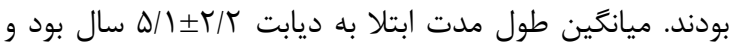

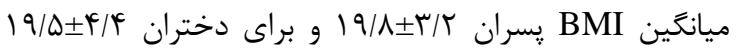

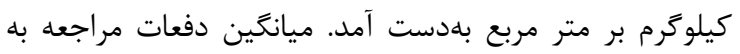

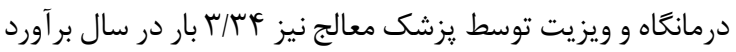

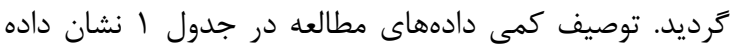

شده است.
از غذا از آنالوى سريعالاثر Aspart با نام تجارى Novorapid

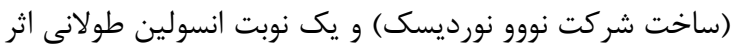

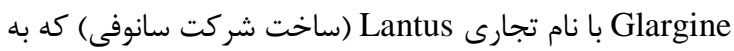
شكل قلم ير شده و با سر سوزن نازكتر در دسترس بود، مورد

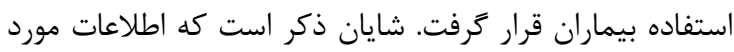

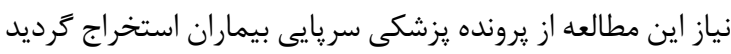

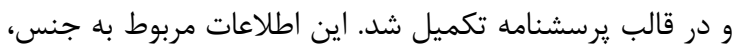

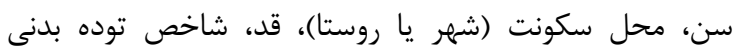

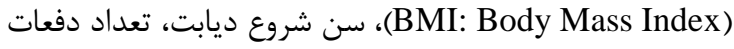

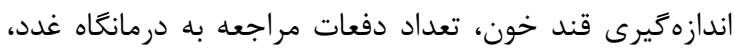

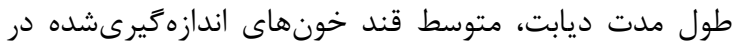

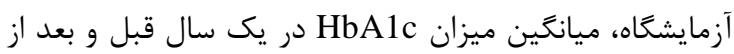
تغيير رزيم درمانى و يك سال قبل و بعد از تغيير رزيم انسولين،

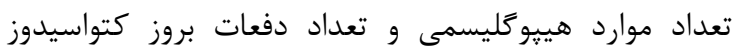

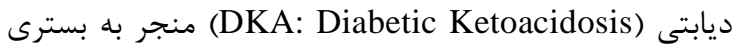
در طول يك سال قبل و بعد از تغيير رزيم انسولين بودند. تعداد دفعات تزريق انسولين، نوع انسولين، نام دستخاه اندازهخيرى

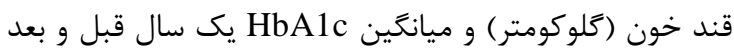

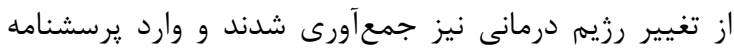
كرديدند. اندازهيرى قند خون بيماران با استفاده از دستخاه

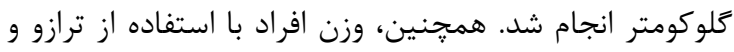
قد ايستاده در بعد از ظهر توسط قدسنج دئن ديوارى سكام اندازهزيرى گرديد. شاخص توده بدنى نيز از طريق تقسيم وزن برن

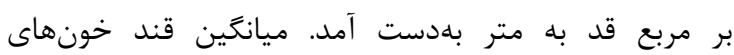

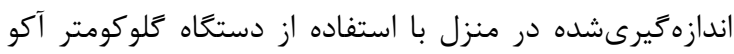

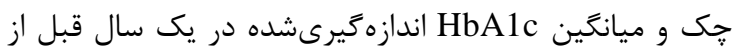
تغيير رزيم درمانى و يك سال بعد از اجراشدن جهار تزريق

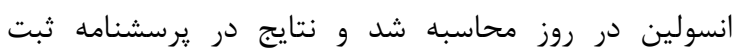

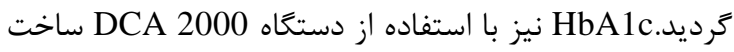

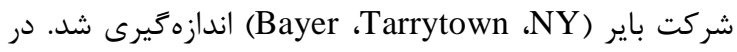
اين مطالعه قند خون كمتر از • • ميلى گرم در دسىليتر بهعنوان

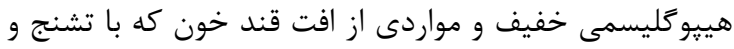

جدول ا: توصيف متغيرهاى كمى بررسىشده در افراد مورد مطالعه

\begin{tabular}{|c|c|c|c|c|}
\hline بيشينه & كمينه & انحراف معيار & ميانگين & \\
\hline 11 & f & $r / \Delta$ & $|1 / 4|$ & سن (سال) \\
\hline 11 & $9 / 4$ & $1 / 4$ & $\Lambda / f$ & HbA1C يك سال قبل HbA1C \\
\hline 1. & 4 & $1 / 1$ & $\Lambda / 1$ & HbA1C يك سال بعد \\
\hline$r \cdot$. & $\wedge \Delta$ & $r V / Q$ & $|F T /|$ & قند خون ناشتا \\
\hline r.. & 10 . & $F V / I$ & $r$ rq/ब & قند خون دو ساعت بعد از غذا \\
\hline$\Delta$ & 1 & $1 / 1$ & r/ & دفعات مراجعه \\
\hline r & • & $1 / 1$ & $1 / 4$ & هيبوَليسمى \\
\hline$r \cdot / \Lambda$ & $1 \cdot / 9$ & $F / l$ & $19 / 9$ & شاخص توده بدنى \\
\hline $1 \cdot$ & 1 & $r / T$ & $\Delta / 1$ & طول ابتلا به ديابت (سال) \\
\hline
\end{tabular}


دفعات مراجعه به يزشك معالج و ميانگين HbA1c ارتباط

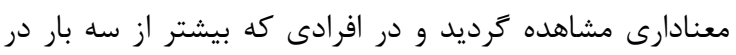

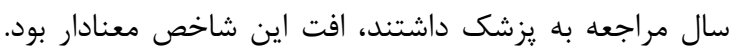
بايد خاطرنشان ساخت كه بين شاخص توده بدنى و ميانگين HbA1C

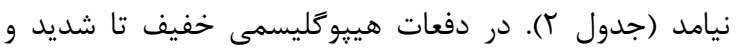

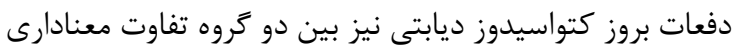

مطابق با نتايج، ميانگين HbA1c قبل از رزيم جهار نوبتى

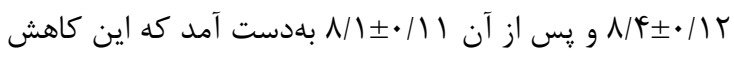

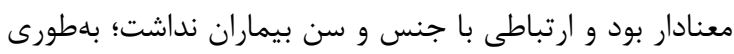

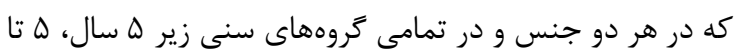

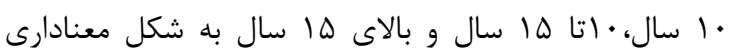

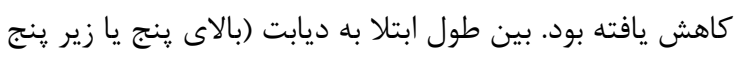

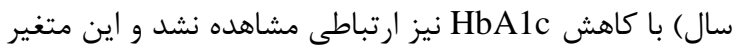
در هر دو گروه بهطور يكسان كاهش يافت اهن از سوى ديكر، بين

جدول r: ارتباط تغييرات HbA1C قبل و بعد از رزيم جهار نوبتى بر حسب متغيرهاى مورد مطالعه

\begin{tabular}{|c|c|c|c|c|c|c|c|}
\hline \multirow{2}{*}{ معنادارى سطح } & \multicolumn{2}{|c|}{ هس از تزريق جهار نوبتى } & \multicolumn{2}{|c|}{ قبل از تزريق جهار نوبتى } & & \multirow{2}{*}{\multicolumn{2}{|c|}{ متغير }} \\
\hline & انحراف معيار & ميانكَين & انحراف معيار & ميانغين & & & \\
\hline$\cdot / \cdot r$ & .111 & N/1 &.$/ 1 T$ & $\Lambda / \uparrow$ & & & HbA1C \\
\hline$\cdot / \cdot \cdot 1$ & $\cdot / 11$ & $V / \Lambda$ & $\cdot|r|$ & $\Lambda / r$ & & يسر & \\
\hline$\cdot / \cdot r$ &.$/ 1 r$ & $\Lambda / r$ & $\cdot / 1 \Delta$ & $N / \Delta$ & & دختر & \\
\hline.$/ \cdot f$ & $\cdot / 1 \pi$ & $\Lambda / 1$ & .119 & $\Lambda / r$ & $\mathrm{n}=91$ & كمتر و مساوى ينج سال & \\
\hline$\cdot 1 \cdot \cdot 1$ & .119 & $1 / 1$ & $.11 \mathrm{~V}$ & $\Lambda / V$ & $n=r q$ & بيش از پينج سال & طول دوره بيمارى \\
\hline .1919 & $\cdot / 10$ & $\Lambda / r$ & .119 & $\Lambda / r$ & $n=r \wedge$ & كمتر و مساوى سه بار & \\
\hline$\cdot 1 \cdot \cdot 1$ & $\cdot 110$ & $V / 9$ & $\cdot / 1 \Lambda$ & N/9 & $\mathrm{n}=\Delta r$ & بيش از سه بار & دفعات مراجعه \\
\hline$\cdot / 499$ &.$/ 1 F$ & $V / 9$ & $\cdot|r|$ & $\Lambda / r$ & $n=r q$ & كمتر و مساوى N/D & \\
\hline$\cdot / \cdot \Lambda$ & $1 / \pi$ & $\Lambda / r$ & $1 / r$ & $\Lambda / 9$ & $n=\Delta 1$ & $\mid \Lambda-r \Delta / \Delta$ & شاخص توده بدنى \\
\hline$\cdot / \cdot 1$ & . & $V / \Delta$ & $\cdot 109$ & $V / 9$ & $\mathrm{n}=1$ & بيش از & \\
\hline$\cdot / \cdot \cdot V$ & $\cdot / \mu r$ & $\Lambda / 1$ & . KT & $1 / 4$ & $n=1$. & كمتر از ينج سال & \\
\hline . & .191 & $V / 9$ & $\cdot / \Lambda V$ & $\Lambda / 1$ & $n=r$. & 11 & \\
\hline$\cdot 1 \cdot \cdot \Delta$ & .119 & $V / \mathcal{F}$ & $\cdot / r \Delta$ & $V / V$ & $n=r \wedge$ & | & \\
\hline$\cdot 1 \cdot \cdot 1$ & $\cdot|\pi|$ & $\mathrm{V} / \cdot$ & $\cdot / 10$ & $V / T$ & $n=r r$ & 11 19 سال & \\
\hline
\end{tabular}

بيشترى را نسبت به انسولينهاى متوسط و كوتاه اثر نشان

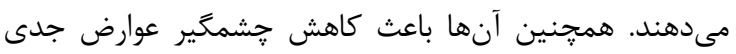

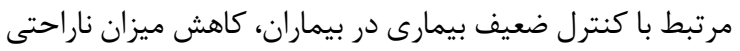

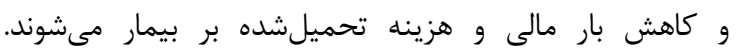

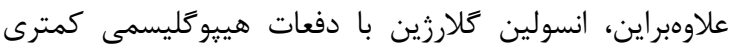

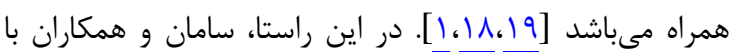

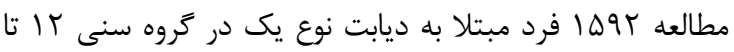

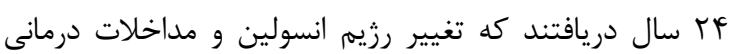

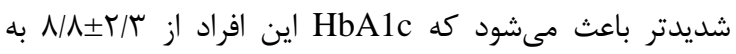

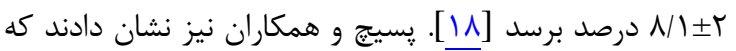

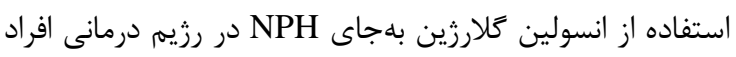

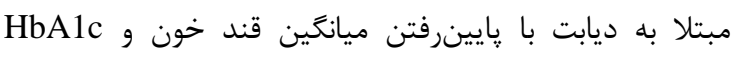

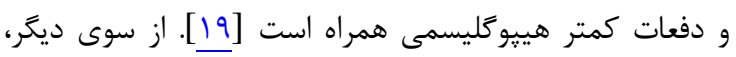

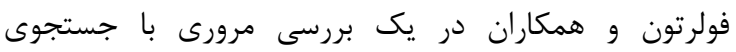

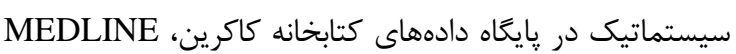

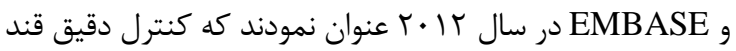

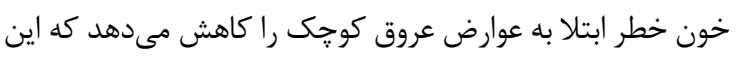

در مطالعه حاضر تأثير دو رزيم مختلف انسوليندرمانى دو

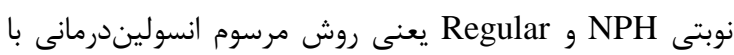
رزيم جهار نوبتى تزريق انسولين در روز Intensive (سه تزريق

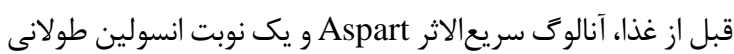

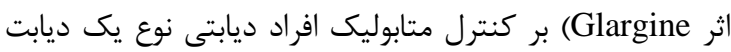

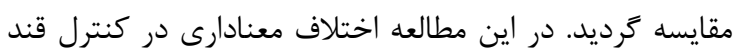

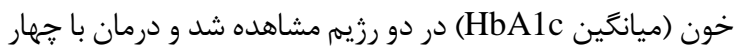

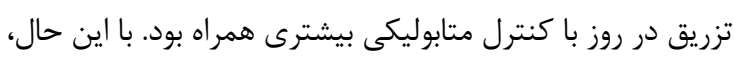

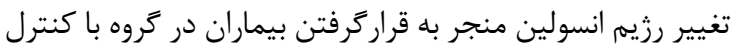

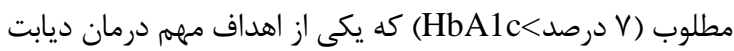

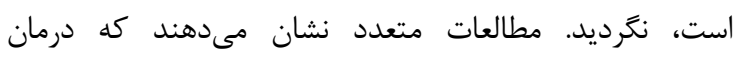

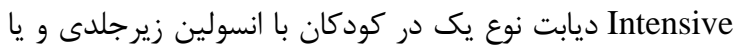

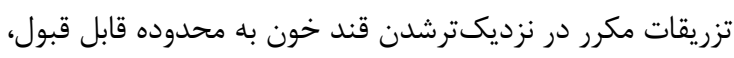

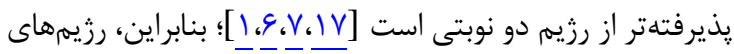

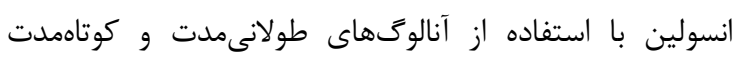

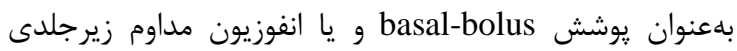

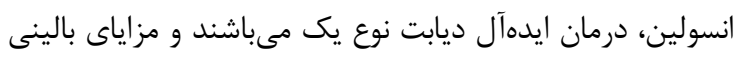


خود را در محدوده طبيعى و يا نزديك به آن نحَه دارند. از سوى

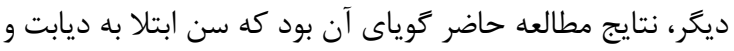

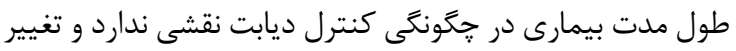
رزيم انسولين بدون توجه به سن باعث بهبود نسبى و كنترل بهرتر

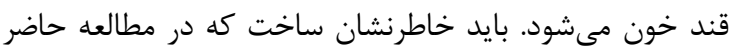
هيج موردى از كتواسيدوز مشاهده نكرديد كه اين امر بيانكر كميليانس خوب بيماران ديابتى در مركز ما مى بـاشد.

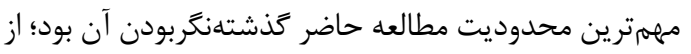

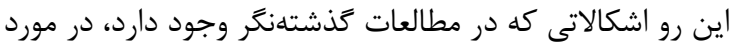
اين مطالعه نيز صدق مى كنند. همجنين در اين مطالئ دالعه ميزان

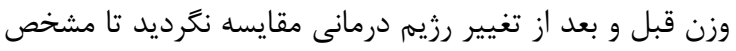

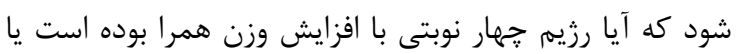
خير؟ به بررسى هزينه دو نوع درمان و ميزان رضايت بيماران و و ونه

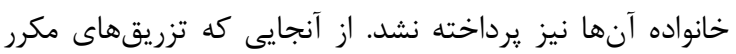

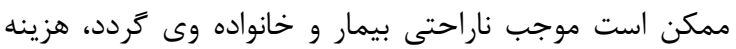
بيشترى داشته و در دسترس همعان نباشد و يا بيمه در تأمين

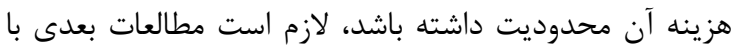

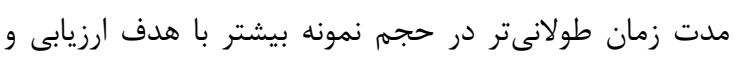
برطرفكردن محدوديتهاى مطالعه حاضر صورت گيرند.

\section{نتيجه تيرى}

يافتههاى مطالعه حاضر نشان داد كه رزيم انسوليندرمانى

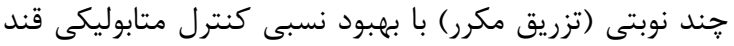

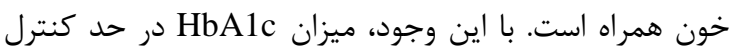

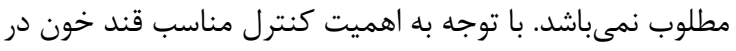
جلوگيرى از عوارض طولانىمدت ديابت، برنامهريزى با تمركز

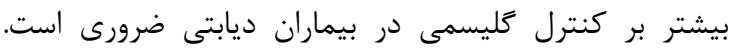

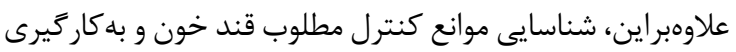
استراترىهاى مناسب براى حذف اين موانع در بيماران تحت هر نوع رزيم درمانى با انسولين ضرورى مى باشد.

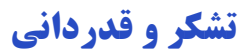

مقاله حاضر بركرفته از ياياننامه دوره دكتراى عمومى

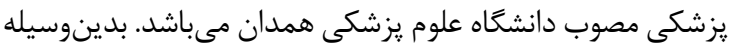

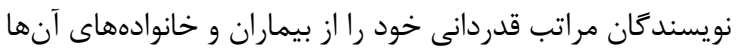
براى همكارى ارزشمندشان در جمعآورى اطلاعات مورد نياز اين

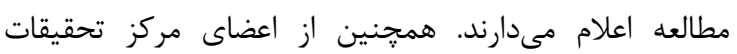

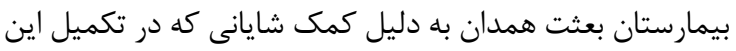

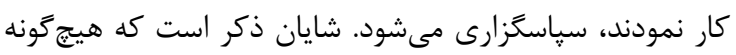
تعارض منافعى در مطالعه حاضر وجود نداشته است.

\section{REFERENCES}

1. Garg S, Moser E, Dain MP, Rodionova A. Clinical experience with insulin glargine in type 1 diabetes. Diabetes Technol Ther. 2010;12(11):835-46. PMID: 20969435 DOI:
اثر عمدتاً در بيماران جوانتر در مراحل اوليه بيمارى مشاهده كرديد [r.

با وجود مزاياى ذكرشده، برخى از صاحبنظران و محققان

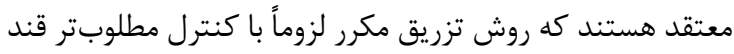

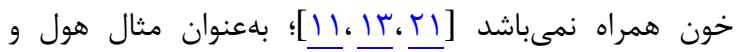

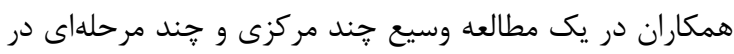

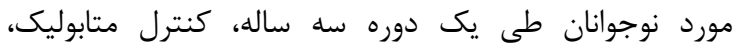

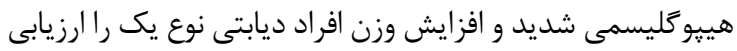

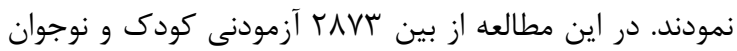

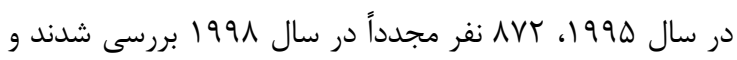

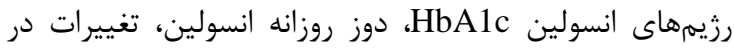

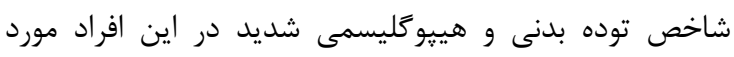
ارزيابى قرار گرفت. بر مبناى نتايج در طول سه سه سال، استفاده از

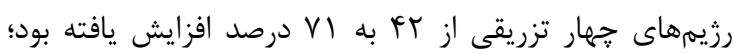

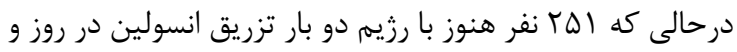

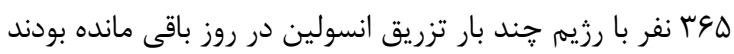

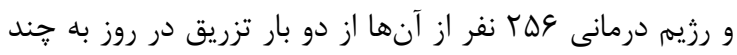

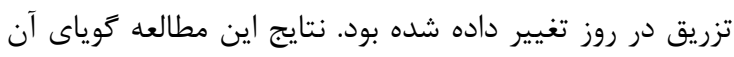

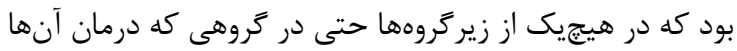

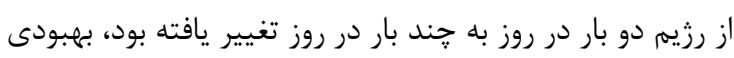

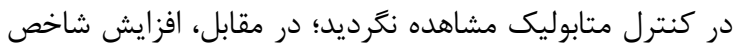

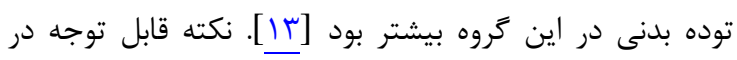

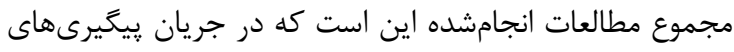

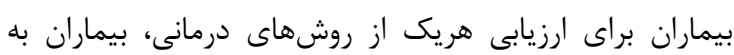

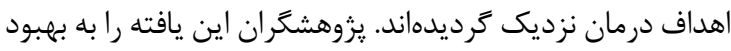

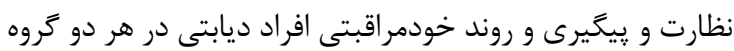
رزيم دو نوبتى (درمان مرسوم) و جهار تزريقى (درمان مطلوب)

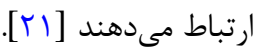
در اين مطالعه ميانخين HbA1c بيماران در حد كندرل

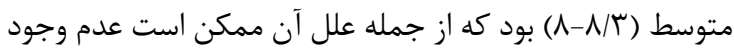

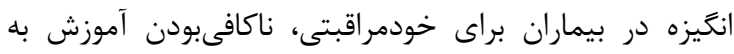

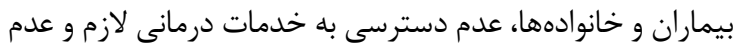

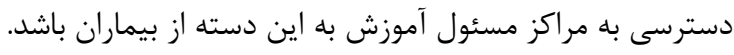

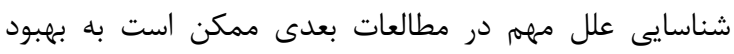

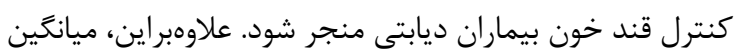

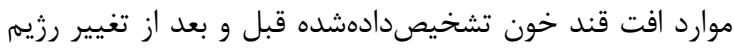

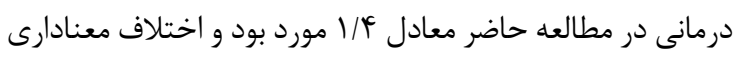
بين دو گروه مشاهده نشد. بايد توجه داشت كه اين يافته قابل

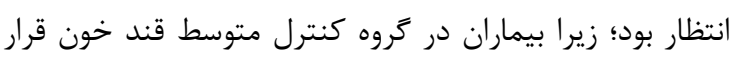

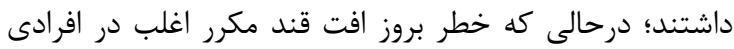

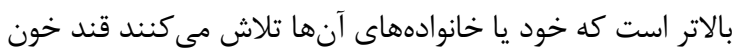

10.1089/dia.2010.0135

2. de Beaufort CE, Swift PG, Skinner CT, Aanstoot HJ, Aman J, Cameron F, et al. Study group on childhood diabetes 2005 
continuing stability of center differences in pediatric diabetes care: do advances in diabetes treatment improve outcome? The Hvidoere Study Group on Childhood Diabetes. Diabetes Care. 2007;30(9):2245-50. PMID: 17540955 DOI: $10.2337 /$ dc07-0475

3. Phelan H, King B, Anderson D, Crock P, Lopez P, Smart C. Young children with type 1 diabetes can achieve glycemic targets without hypoglycemia: results of a novel intensive diabetes management program. Pediatr Diabetes. 2018; 19(4):769-75. PMID: 29504243 DOI: 10.1111/pedi.12644

4. Ludvigsson J, Bolli GB. Intensive insulin treatment in diabetic children. Diabetes Nutr Metab. 2001;14(5):292-304. PMID:11806471

5. Ratner RE, Hirsch IB, Neifing JL, Garg SK, Mecca TE, Wilson CA. Less hypoglycemia with insulin glargine in intensive insulin therapy for type 1 diabetes. U.S. study group of insulin glargine in type 1 diabetes. Diabetes Care. 2000;23(5):639-43. PMID: 10834423

6. Hanaire-Broutin $H$, Melki $V$, Bessières-Lacombe $S$, Tauber JP. Comparison of continuous subcutaneous insulin infusion and multiple daily injection regimens using insulin lispro in type 1 diabetic patients on intensified treatment: a randomized study. The study group for the development of pump therapy in diabetes. Diabetes Care. 2000;23(9):12325. PMID: 10977011

7. Tricco AC, Ashoor HM, Antony J, Beyene J, Veroniki AA, Isaranuwatchai $\mathrm{W}$, et al. Safety, effectiveness, and cost effectiveness of long acting versus intermediate acting insulin for patients with type 1 diabetes: systematic review and network meta-analysis. BMJ. 2014;349:g5459. PMID: 25274009

8. Lalli C, Ciofetta M, Del Sindaco P, Torlone E, Pampanelli S, Compagnucci P, et al. Long-term intensive treatment of type 1 diabetes with the short-acting insulin analog lispro in variable combination with $\mathrm{NPH}$ insulin at meal time. Diabetes Care. 1999;22(3):468-77. PMID: 10097931

9. Nordwall M, Arnqvist HJ, Bojestig M, Ludvigsson J. Good glycemic control remains crucial in prevention of late diabetic complications- the linkoping diabetes complications study. Pediatr Diabetes. 2009;10(3):168-76. PMID: 19175900 DOI: 10.1111/j.1399-5448.2008.00472.x

10. Machado-Alba JE, Medina-Morales DA. Comparison of the impact of human vs analogue insulins on glycosylated haemoglobin in a population with diabetes mellitus. Int $J$ Clin Pract. 2016;70(12):996-1002. PMID: 28032423 DOI: 10.1111/ijcp.12904

11. Ruospo M, Saglimbene VM, Palmer SC, De Cosmo S, Pacilli A, Lamacchia O, et al. Glucose targets for preventing diabetic kidney disease and its progression. Cochrane Database Syst Rev. 2017;6:CD010137. PMID: 28594069 DOI: 10.1002/ 14651858.CD010137.pub2

12. O'Hagan M, Harvey JN; Brecon Group. Glycemic control in children with type 1 diabetes in wales: influence of the pediatric diabetes specialist nurse. Diabetes Care. 2010;33(8):1724-6. PMID: 20435792 DOI: 10.2337/dc092304

13. Holl RW, Swift PG, Mortensen HB, Lynggaard H, Hougaard $\mathrm{P}$, Aanstoot $\mathrm{HJ}$, et al. Insulin injection regimens and metabolic control in an international survey of adolescents with type 1 diabetes over 3 years: results from the Hvidore study group. Eur J Pediatr. 2003;162(1):22-9. PMID: 12486503 DOI: 10.1007/s00431-002-1037-2

14. Razavi Z, Maher S, Fredmal J. Comparison of subcutaneous insulin aspart and intravenous regular insulin for the treatment of mild and moderate diabetic ketoacidosis in pediatric patients. Endocrine. 2018;61(2):267-74. PMID: 29797212 DOI: 10.1007/s12020-018-1635-z

15. Lone SW, Siddiqui EU, Muhammed F, Atta I, Ibrahim MN, Raza J. Frequency, clinical characteristics and outcome of diabetic ketoacidosis in children with type-1 diabetes at a tertiary care hospital. J Pak Med Assoc. 2010;60(9):725-9. PMID: 21381577

16. Rewers M, Pihoker C, Donaghue K, Hanas R, Swift P, Klingensmith GJ. Assessment of glycemic control and adolescents with diabetes. Pediatr Diabetes. 2009;10:71-81. DOI: 10.1111/j.1399-5448.2009.00582.x

17. Aschner P, Horton E, Leiter LA, Munro N, Skyler JS; Global partnership for effective diabetes management. Practical steps to improving the management of type 1 diabetes: recommendations from the global partnership for effective diabetes management. Int J Clin Pract. 2010;64(3):305-15. PMID: 20456170 DOI: 10.1111/j.1742-1241.2009.02296.X

18. Sämann A, Lehmann T, Kloos C, Braun A, Hunger-Dathe W, Wolf $\mathrm{G}$, et al. Flexible, intensive insulin therapy and dietary freedom in adolescents and young adults with Type 1 diabetes: a prospective implementation study. Diabet Med. 2008;25(5):592-6. PMID: 18445173 DOI: 10.1111/j.14645491.2008.02406.x

19. Pesić M, Zivić S, Radenković S, Velojić M, Dimić D, Antić $\mathrm{S}$.Comparison between basal insulin glargine and NPH insulin in patients with diabetes type 1 on conventional intensive insulin therapy. Vojnosanit Pregl. 2007;64(4):24752. PMID: 17580534

20. Fullerton B, Jeitler K, Seitz M, Horvath K, Berghold A, Siebenhofer A. Intensive glucose control versus conventional glucose control for type 1 diabetes mellitus. Cochrane Database Syst Rev. 2014;2:CD009122. PMID: 24526393 DOI: 10.1002/14651858.CD009122.pub2

21. Rostami P, Setoodeh A, Rabbani A Nakhaei-Moghadam M, Varzaneh N, Rezaei N. A randomized clinical trial of insulin glargine and aspart, compared to NPH and regular insulin in children with Type 1 diabetes mellitus Iranian. Iran J Pediatr. 2014;24(2):173-8. PMID: 25535536 